\title{
Estrategia feedback en el desarrollo de habilidades investigativas de estudiantes universitarios
}

\section{Kutipay kamachikuykuna ashiy yaćhaykuna wiñachininćhu hatun yaćhaywasip yaćhapakukunap}

Recepción: 16 junio 2020 Corregido: 29 septiembre 2020 Aprobación: 01 noviembre 2020

Pedro Huauya Quispe

Nacionalidad: Peruano / Universidad Nacional de San Cristóbal de Huamanga Correo: pedro.huauya@unsch.edu.pe / ORCID: https://orcid.org/oooo-0003-0156-2622

Víctor Alcides Coaquira Cárdenas

Nacionalidad: Peruano / Universidad Nacional de San Cristóbal de Huamanga Correo: Victor.coaquira@unsch.edu.pe / ORCID: https://orcid.org/oooo-0003-0108-8369

Edison Laderas Huillcahuri

Nacionalidad: Peruano / Universidad Nacional de San Cristóbal de Huamanga Correo:edison.laderas@unsch.edu.pe / ORCID: https://orcid.org/oooo-ooo2-4598-319X

\section{Resumen}

Tiene por objetivo de analizar las incidencias de la estrategia feedback en el desarrollo de habilidades investigativas de los estudiantes de educación primaria y secundaria de la Facultad de Ciencias de la Educación, 2019; nivel de investigación explicativa de diseño pre experimental con pre y postest, la muestra constituyó 39 estudiantes de educación primaria y secundaria, los datos fueron recolectado a través de la técnica de encuesta y prueba pedagógica; se realizó la prueba de hipótesis a través de $t$ de Student para muestras relacionadas con un nivel de confianza de $95 \%$. Se concluye que al $95 \%$ de nivel de confianza, la estrategia feedback incide significativamente en el desarrollo de habilidades investigativas de los estudiantes de educación primaria y secundaria de la Facultad de Ciencias de la Educación, $2019(0,000<0,05)$.

\section{Palabras clave:}

Educación, feedback, desarrollo, habilidades, investigación

\section{Lisichiku limaykuna:} yaćhachina, Kutipay, wiñay, atipaykuna, ashipay 


\title{
Feedback Strategy in the Development of Research skills of University Students
}

\begin{abstract}
Its objective is to analyze the incidences of the feedback strategy in the development of investigative skills of students of primary and secondary education of the Faculty of Education Sciences, 2019; level of explanatory research of pre-experimental design with pre and post-test, the sample consisted of 39 students from primary and secondary education, the data was collected through the survey technique and pedagogical test; The hypothesis test was carried out through Student's t for related samples with a confidence level of $95 \%$. It is concluded that at $95 \%$ confidence level, the feedback strategy significantly affects the development of investigative skills of students of primary and secondary education of the Faculty of Education Sciences, 2019 (0.000 <0.05).
\end{abstract}

\author{
Keywords \\ Education, feedback, \\ development, skills, \\ research
}

\section{Estratégia de feedback no desenvolvimento de habilidades investigativas de estudantes universitários}

\section{Resumo}

Seu objetivo é analisar a incidência da estratégia de feedback no desenvolvimento de habilidades investigativas nos estudantes do Curso de Ciências da Educação para Ensino fundamental e médio 2019; nível de pesquisa explicativa de desenho pré-experimental com pré e pós-teste, a amostra constituiu-se de 39 estudantes do ensino fundamental e médio, os dados foram coletados por meio da técnica de enquete e teste pedagógico; $O$ teste de hipótese foi realizado por meio do teste t de Student para amostras relacionadas com nível de confiança de $95 \%$. Conclui-se que, em um nível de confiança de $95 \%$, a estratégia de feedback incide significativamente no desenvolvimento de habilidades investigativas nos estudantes do Curso de Ciências da Educação para Ensino fundamental e médio 2019 (0,000 <0,05).

\section{Datos de los autores}

Pedro Huauya Quispe, es docente e investigador en Educación y Educación matemática, Maestro en Estrategia de Enseñanza, Aprendizaje y Evaluación por la Universidad Nacional de San Cristóbal de Huamanga, Doctor en Ciencias de la Educación por la Universidad Nacional de Educación.

Víctor Alcides Coaquira Cárdenas, es docente e investigador en el área Educación matemática, Maestro en Docencia Universitaria por la Universidad Nacional de San Cristóbal de Huamanga, estudiante del Doctorado en Matemática en la Universidad Nacional del Santa Chimbote-Perú.

Edison Laderas Huillcahuari, es docente e investigador en el área Educación matemática, Maestro en Docencia Universitaria por la Universidad Nacional de San Cristóbal de Huamanga, estudiante del Doctorado en Matemática en la Universidad Nacional del Santa Chimbote-Perú. Segunda especialidad en Ingeniería Civil. 


\section{Introducción}

En el mundo actual globalizado del siglo XXI, sociedad del conocimiento y la posmodernidad, la investigación es de vital importancia en la formación profesional de los estudiantes universitarios, articulado con el perfil de egreso y fines de la universidad.

Actualmente la naturaleza social y compleja de la educación universitaria y los desafíos de calidad que exige el mercado laboral; la investigación es la vía necesaria para el desarrollo de capacidades que permiten abordar con eficiencia y eficacia los cambios que producen los avances de la ciencia y la tecnología; así como el mejoramiento profesional y su contribución al desarrollo socioeconómico de la región y del país.

En particular, la Universidad Nacional de San Cristóbal de Huamanga se encuentra en $15,3 \%$, internacionalización 11,1\%, índice de investigación $0,7 \%$, índice de empleabilidad registra 9,5\%, prestigio $8,5 \%$. Mientras que el número de profesores supera los 500 , los estudiantes llegan a los 11 mil y las publicaciones científicas hasta el año 2013 eran solo siete (Flores, 2015), resultado que refleja escasa producción intelectual y difusión de los artículos cientíicos en las revistas indizadas de trascendencia internacional. Implica que existe el desinterés del estado en la política investigativa y en cada la universidad con excesos horas de clase, con menor cantidad de docentes principales y asociados, planes de estudio desfasados que no permiten promover y formar estudiantes investigadores, los cuales demuestran el escaso desarrollo de habilidades investigativas de los estudiantes universitarios a nivel de pregrado y posgrado.

Según la Ley universitaria $\mathrm{N}^{\circ} 30220$ para obtener el grado académico y título profesional es necesario realizar la investigación. Lo cual implica, desarrollar las habilidades investigativas de los estudiantes universitarios, sin embargo, existe una marcada diferencia de dificultades en el desarrollo de las habilidades investigativas de los estudiantes y dominio de la metodología de investigación de los docentes.

El desarrollo de las habilidades investigativas en el pregrado constituye un factor muy importante para la investigación científica. Una de las tendencias fundamentales es la correlación entre la formación de habilidades para la investigación o desarrollo de habilidades investigativas y formación para la investigación (Martínez y Márquez, 2014),

Pérez y López (1999, citado por Martínez y Márquez, 2014) definen que las habilidades investigativas son acciones psíquicas y prácticas que permiten la regulación racional de la actividad de la actividad académica.

Machado y Montes de Oca (2009, citado por Martínez y Márquez, 2014) el desarrollo de habilidades investigativas es una de las vías que permite integrar el conocimiento, a la vez sirve como sustento de autoaprendizaje constante; no solo facilitan la solución de las diversas contradicciones que surgen en el ámbito laboral y científico, sino además, permiten la autocapacitación permanente y la actualización sistemática de los conocimientos, lo cual es un indicador de competitividad en la época moderna.

Según Fiallo (2001, citado por Martínez y Márquez, 2014) que las habilidades investigativas es un eje transversal para responder a las necesidades sociales de cada momento histórico concreto, exige el análisis y remodelación de los ejes establecidos en correspondencia con las necesidades sociales futuras. 
Según Aguilar et al. (2009) para lograr habilidades investigativas es importante que los profesionales posean destreza para transmitir una correcta estructura del pensamiento, claridad con el estilo de escribir en el arreglo espacial de las palabras, mientras presenten la información escrita sin defectos de ortografía o sintaxis. Para la comunicación científica necesitan además la capacidad de síntesis y congruencia en la redacción de los elementos metodológicos, como lo son los objetivos, las hipótesis o conclusiones. Para ello, la evaluación de protocolos escritos por los estudiantes de pregrado es una vía útil para evaluar y reforzar las habilidades comunicativas de los estudiantes en formación, perfeccionar capacidades profesionales críticas y facilitar su razonamiento científico metodológico.

Paracontribuirenlasolucióndelasituación problemáticadel presentetrabajodeinvestigación se plantó los objetivos, analizar la incidencia de la estrategia feedback en el desarrollo de habilidades investigativas de los estudiantes de educación primaria y secundaria de la Facultad de Ciencias de la Educación, 2019, determinar las influencias de la estrategia feedback en el desarrollo de habilidades cognitivas, instrumentales y habilidades afectivas de los estudiantes universitarios.

El feedback o la retroalimentación es una buena estrategia de aprendizaje que sirve principalmente para desarrollar las habilidades investigativas de los estudiantes universitarios, ya es un trabajo individualizado y colectivo. Es una estrategia que permite permanentemente realizar el seguimiento, orientación, monitorear el proceso, y revisar los resultados obtenidos en un período específico. Su propósito es generar aprendizajes que permitan la mejora continua del rendimiento individual, y entregar elementos relevantes para el desarrollo de las habilidades investigativas.

Para Jiménez (2015), desde el enfoque constructivista sociocultural y situado, el feedback es una ayuda potencial del profesor al estudiante, que se aplica a lo largo del proceso de enseñanza y aprendizaje. El feedback deber ser descriptivo, simple y objetivamente claro y centrado sobre la actividad o tarea concreta, en cuyo caso disminuye la carga emocional del estudiantado dado que se representa la situación como manejable.

Según Mamani (2018), el feedback es un término en inglés, traducido en español retroalimentación. El feedback se da de dos formas uno intrínseco y extrínseco, de modo que contribuye a individualizar el aprendizaje, diagnosticar dificultades, constatar la competencia aprendizaje, orientar a corregir y motivar al estudiante.

Para Aguilar et al. (2009), la retroalimentación provee al estudiante reflexión sobre sus actos y consecuencias, le permite llegar a las metas y objetivos y se considera un medio para fortalecer el proceso de aprendizaje y desempeño docente. La retroalimentación constructiva se caracteriza por ser: pertinente, inmediata, factual, útil, confidencial, respetuosa, adaptada y alentadora.

Según Calderón (2010) El feedback es un mecanismo importante que aumenta el valor de algunas reglas de actuación y disminuye el valor de otras. La retroalimentación llega al cerebro a partir de dos inicios diferentes desde e entorno, por medio de los efectos sobre el cuerpo y desde la comunicación intencional de un maestro. 


\section{Materiales y métodos}

El presente trabajo de investigación es de tipo de investigación cuantitativa aplicada de nivel explicativa con diseño pre experimental con pre y postest. La muestra constituyó 39 estudiantes de la serie 500 de la escuela profesional de educación primaria y secundaria, los datos se recolectaron a través de la técnica de observación y la prueba pedagógica sobre el desarrollo de las habilidades investigativas. La validación de los instrumentos de recolección de datos se realizó a través de juicio de 3 expertos y la fiabilidad con la prueba de Alpha de Cronbach; los datos se procesaron a través de la estadística descriptiva y la prueba de hipótesis se realizó con la prueba paramétrica T de Student para muestras relacionadas con 95\% de nivel de confianza, previa comprobación de la normalidad de los datos. El área de estudio constituyó los estudiantes de la escuela profesional de educación primaria y secundaria de la Facultad de Ciencias de la Educación de la Educación de la Universidad Nacional de San Cristóbal de Huamanga en el periodo lectivo del semestre académico 2019- I y II.

Los procesos de retroalimentación se realizaron de manera permanente en el dictado de la asignatura de investigación en cuarto etapas: acciones de hacer sugerencia, acciones de clarificar, valorar, expresar inquietudes y hacer sugerencias. En la primera etapa se realizó conversaciones y preguntas acerca de las debilidades de aprendizaje tanto cognitivas, habilidades y actitudes con la finalidad de clarificar y realizar el reforzamiento; segunda etapa, se destacó y se valoró las fortalezas y los aspectos positivos del logro de aprendizaje y trabajo académico generando clima de confianza; tercera etapa, se realizó actividades seguimiento y acompañamiento a través de las interrogantes, en base de sus inquietudes hasta lograr con los propósitos del aprendizaje y desarrollo de las habilidades investigativas; finalmente en la cuarta etapa, se realizó las sugerencias correspondiente sobre como resolver dificultades y problemas que se presentan en el proceso de aprendizaje y desarrollo de las habilidades investigativas.

Tabla 1

Escala de Valoración de las Habilidades Investigativas

\begin{tabular}{llc}
\hline \multicolumn{1}{c}{ Categorías } & \multicolumn{1}{c}{ Descripción } & Código \\
\hline En inicio [00- 05] & $\begin{array}{l}\text { No evidencia desarrollo de las habilidades } \\
\text { investigativas }\end{array}$ & 1 \\
Proceso [06-10] & $\begin{array}{l}\text { Evidencia parcialmente el desarrollo de las habilidades } \\
\text { investigativas }\end{array}$ & 2 \\
Satisfactorio [11-15] & $\begin{array}{l}\text { Evidencia lo previsto el desarrollo de las habilidades } \\
\text { investigativas }\end{array}$ & 3 \\
Excelente [16-20] & $\begin{array}{l}\text { Evidencia con excelencia el desarrollo de las } \\
\text { habilidades investigativas }\end{array}$ & 4 \\
\hline
\end{tabular}

Fuente. Elaboración propia 


\section{Resultados}

Figura 1

Habilidades Cognitivas de los Estudiantes

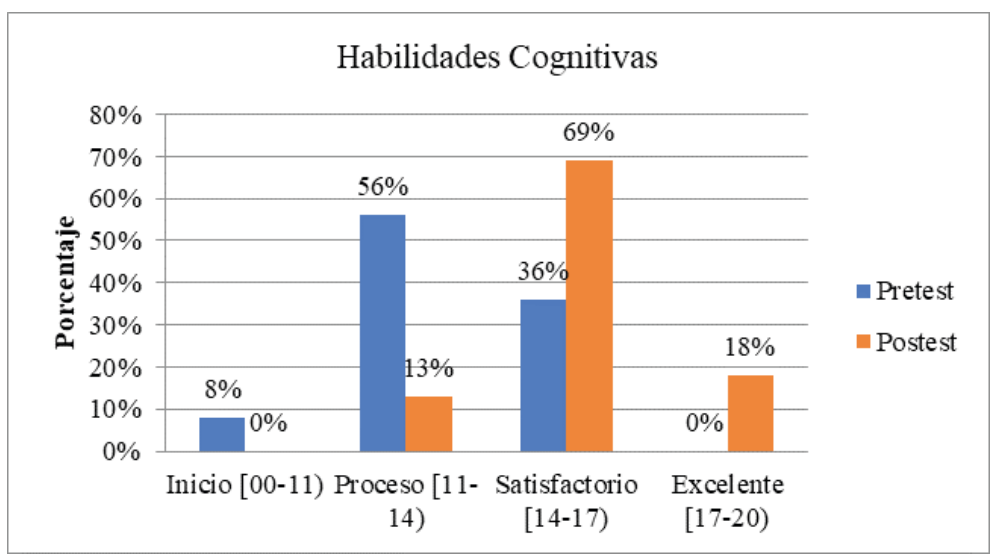

Fuente. Datos de la ficha de observación a los estudiantes, 2019

En la figura 1 se observa, que antes de la aplicación de la estrategia fuedback, en el pretest, el $8 \%$ de los estudiantes se encontraba en nivel inicio en el manejo de habilidades investigativas cognitivas, $56 \%$ en proceso y $36 \%$ satisfactorio. Mientras que después de la aplicación de la estrategia feedback, el o\% de los estudiantes lograron un nivel inicio en el manejo de habilidades investigativas cognitivas, $13 \%$ proceso, $69 \%$ satisfacción y $18 \%$ excelente.

Por consiguiente, con la aplicación de la estrategia de feedback se evidencia una diferencia significativa en el manejo de habilidades investigativas cognitivas. Los estudiantes lograron el manejo de la búsqueda de información a través de libros físicos y digitales, revistas científicas electrónicas, distingue evidencias científicas de otro tipo de evidencias empíricas y toma posición del enfoque teórico. Logró con satisfacción el manejo metodológico de la investigación, en el planteamiento del problema, realizar diseño metodológico contextualizado según el enfoque de la investigación y discusión de resultados y conclusión científica en el trabajo.

\section{Figura 2}

Habilidades Instrumentales de los Estudiantes

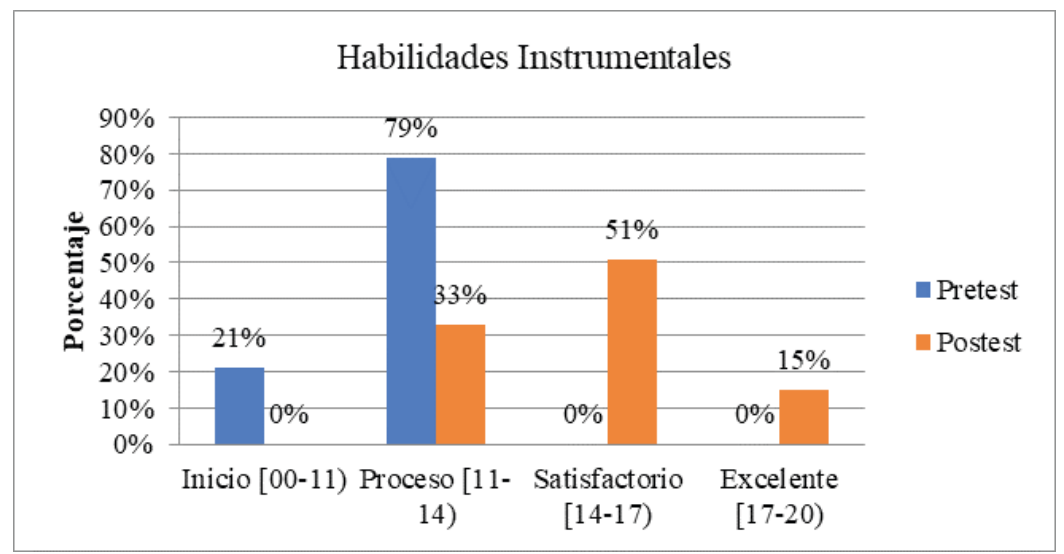

Fuente. Datos de la ficha de observación a los estudiantes, 2019 
En la figura 2 se observa, en el pretest, el $21 \%$ de los estudiantes se encontraba en nivel inicio en el manejo de habilidades instrumentales y $79 \%$ en proceso. Mientras que después de la aplicación de la estrategia fuedback, el o\% de los estudiantes lograron un nivel inicio en el manejo de habilidades instrumentales, 33\% proceso, 51\% satisfacción y 15\% excelente.

Por consiguiente, con la aplicación de la estrategia de feedback se evidencia una diferencia significativa en el desarrollo de las habilidades instrumentales. Los estudiantes lograron manejo óptimo de la tecnológico de la ofimática el software SPSS, comunicación escrita según normas internacionales de redacción y comunicación oral.

Figura 3

Habilidades Afectivas de los Estudiantes

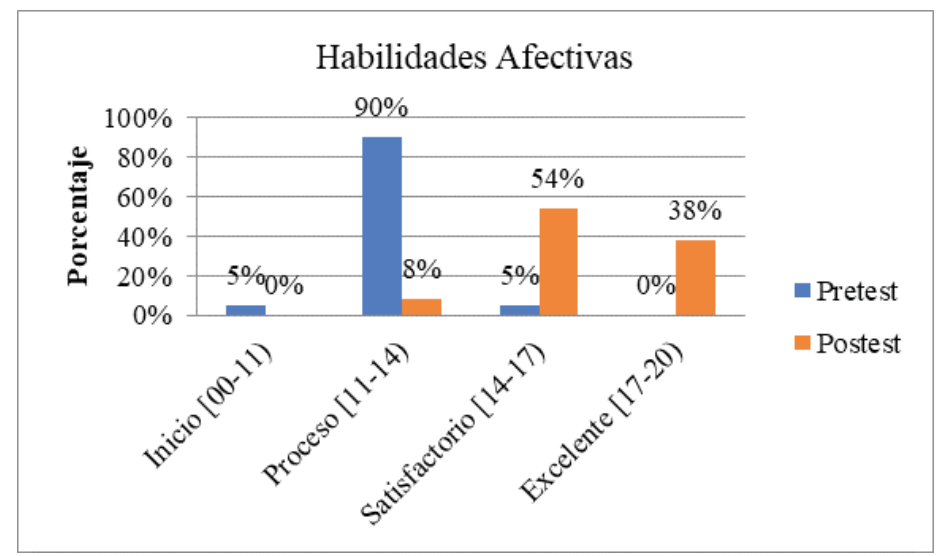

Fuente. Datos de la ficha de observación a los estudiantes, 2019

En la figura 3 se observa, en el pretest el $5 \%$ de los estudiantes se encontraba en nivel inicio en el manejo de habilidades afectivas, $90 \%$ en proceso y $5 \%$ en satisfactorio. Mientras que después de la aplicación de la estrategia feedback, el o\% de los estudiantes lograron un nivel inicio en el manejo de habilidades afectivas, $8 \%$ proceso, $54 \%$ satisfacción y $38 \%$ excelente.

Por consiguiente, con la aplicación de la estrategia de feedback se evidencia una diferencia significativa en el desarrollo de las habilidades investigativas de los estudiantes. Mayor porcentaje de los estudiantes lograron satisfactoria y excelente en el manejo de trabajo en equipo, aprendizaje colaborativo, responsabilidad e interés de aprendizaje en los temas de investigación científica.

Tabla 2

Desarrollo de Habilidades Investigativas

\begin{tabular}{lcccc}
\hline \multirow{2}{*}{ Categoría } & \multicolumn{2}{c}{ Pretest } & \multicolumn{2}{c}{ Postest } \\
\cline { 2 - 5 } & $\mathrm{f}$ & $\%$ & $\mathrm{f}$ & $\%$ \\
\hline Inicio [00-11) & 2 & 5 & 4 & 0 \\
Proceso [11-14) & 27 & 69 & 26 & 67 \\
Satisfactorio [14-17) & 10 & 26 & 9 & 23 \\
Excelente [17-20) & 0 & 0 & 39 & 100 \\
\hline Total & 39 & 100 & 0 \\
\hline
\end{tabular}

Fuente. Datos de la ficha de observación a los estudiantes, 2019 
En la tabla 2 se observa, en el pretest, el $5 \%$ de los estudiantes se encontraba en nivel inicio en el desarrollo de habilidades investigativas, $69 \%$ en proceso y $26 \%$ en satisfactorio. Mientras que después de la aplicación de la estrategia feedback, el o\% de los estudiantes lograron un nivel inicio en el desarrollo de habilidades investigativas, $10 \%$ proceso, $67 \%$ satisfacción y $23 \%$ excelente.

\section{Figura 4}

Desarrollo de Habilidades Investigativas de los Estudiantes

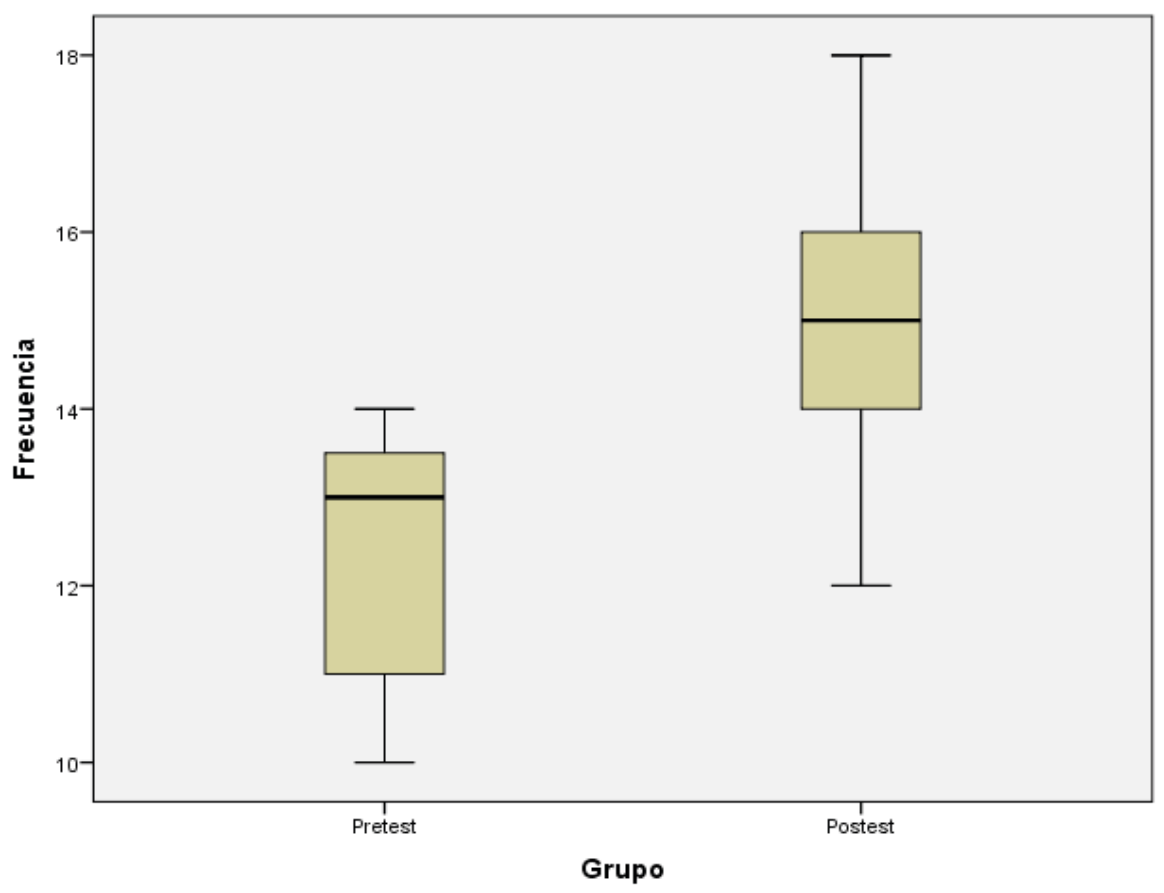

Fuente. Datos de la ficha de observación a los estudiantes, 2019

Según la figura 4 en el pretest, el $25 \%$ de los estudiantes lograron una calificación menores o iguales a $11,50 \%$ menores o iguales a 13 y $75 \%$ menores o iguales a 14 . Mientras que con la aplicación de la estrategia de feedback, el $25 \%$ de los estudiantes lograron calificaciones menores o iguales a 14 en el desarrollo de habilidades investigativas, 50\% menores o iguales a 15 y $75 \%$ menores o iguales a 16

Existe evidencia una diferencia significativa en el desarrollo de las habilidades investigativas. Es decir, los estudiantes lograron mayor desarrollo de habilidades cognitivas, instrumentales y afectivas. 
Tabla 3

Resultado de la Prueba de Hipótesis

\begin{tabular}{|c|c|c|c|}
\hline Hipótesis & $\begin{array}{l}\text { Significancia } \\
\text { calculada }\end{array}$ & $\begin{array}{l}\text { Significancia } \\
\text { asumida }\end{array}$ & Decisión al 95\% de nivel de confianza \\
\hline General & \multirow{4}{*}{ ०,००० (०\%) } & \multirow{4}{*}{$0,05(5 \%)$} & $\begin{array}{l}\text { La estrategia feedback incide significativamente en el } \\
\text { desarrollo de habilidades investigativas de los estudiantes } \\
\text { de educación primaria y secundaria de la Facultad de } \\
\text { Ciencias de la Educación, 2019. }\end{array}$ \\
\hline $\begin{array}{c}\text { Específico } \\
1\end{array}$ & & & $\begin{array}{l}\text { La estrategia feedback influye significativamente en el } \\
\text { desarrollo de las habilidades cognitivas de los estudiantes. }\end{array}$ \\
\hline $\begin{array}{c}\text { Específico } \\
2\end{array}$ & & & $\begin{array}{l}\text { La estrategia feedback influye significativamente en } \\
\text { el desarrollo de las habilidades instrumentales de los } \\
\text { estudiantes. }\end{array}$ \\
\hline $\begin{array}{c}\text { Específico } \\
3\end{array}$ & & & $\begin{array}{l}\text { La estrategia feedback influye significativamente en el } \\
\text { desarrollo de las habilidades afectivas de los estudiantes. }\end{array}$ \\
\hline
\end{tabular}

Fuente. Datos de la ficha de observación a los estudiantes, 2019

\section{Discusión de resultados}

Al 95\% de nivel de confianza, la estrategia feedback incide significativamente en el desarrollo de habilidades investigativas de los estudiantes de educación primaria y secundaria de la Facultad de Ciencias de la Educación, 2019 (0,000<0,05). Existe diferencia significativa en el desarrollo de habilidades investigativas, cognitivas, instrumentales y afectivas.

Para Jiménez (2015), desde el enfoque constructivista sociocultural y situado, el feedback es una ayuda potencial del profesor al estudiante, que se aplica a lo largo del proceso de enseñanza y aprendizaje. El feedback deber ser descriptivo, simple y objetivamente claro y centrado sobre la actividad o tarea concreta, en cuyo caso disminuye la carga emocional del estudiantado dado que se representa la situación como manejable.

Según Mamani (2018), el feedback es un término en inglés, traducido en español retroalimentación. El feedback se da de dos formas uno intrínseco y extrínseco, de modo que contribuye a individualizar el aprendizaje, diagnosticar dificultades, constatar la competencia aprendizaje, orientar a corregir y motivar al estudiante.

Según Fiallo (2001, citado por Martínez y Márquez, 2014) que las habilidades investigativas es un eje transversal para responder a las necesidades sociales de cada momento histórico concreto, exige el análisis y remodelación de los ejes establecidos en correspondencia con las necesidades sociales futuras.

Según Aguilar et al. (2009) para lograr habilidades investigativas es importante que los profesionales posean destreza para transmitir una correcta estructura del pensamiento, claridad con el estilo de escribir en el arreglo espacial de las palabras, mientras presenten la información escrita sin defectos de ortografía o sintaxis. Para la comunicación científica necesitan además la capacidad de síntesis y congruencia en la redacción de los elementos metodológicos, como lo son los objetivos, las hipótesis o conclusiones. Para ello, la evaluación de 
protocolos escritos por los estudiantes de pregrado es una vía útil para evaluar y reforzar las habilidades comunicativas de los estudiantes en formación, perfeccionar capacidades profesionales críticas y facilitar su razonamiento científico metodológico.

Al 95\% del nivel de confianza la estrategia feedback influye significativamente en el desarrollo de las habilidades cognitivas de los estudiantes $(0,00<0,05)$. Existe diferencia significativa en el manejo de habilidades investigativas cognitivas. Los estudiantes lograron el manejo de la búsqueda de información a través de libros físicos y digitales, revistas científicas electrónicas, distingue evidencias científicas de otro tipo de evidencias empíricas y toma posición del enfoque teórico. Logró con satisfacción el manejo metodológico de la investigación, en el planteamiento del problema, realizar diseño metodológico contextualizado según el enfoque de la investigación y discusión de resultados y conclusión científica en el trabajo.

Para Aguilar et al. (2009), la retroalimentación provee al estudiante reflexión sobre sus actos y consecuencias, le permite llegar a las metas y objetivos y se considera un medio para fortalecer el proceso de aprendizaje y desempeño docente. La retroalimentación constructiva se caracteriza por ser: pertinente, inmediata, factual, útil, confidencial, respetuosa, adaptada y alentadora.

Pérez y López (1999, citado por Martínez y Márquez, 2014) definen que las habilidades investigativas son acciones psíquicas y prácticas que permiten la regulación racional de la actividad de la actividad académica.

Machado y Montes de Oca (2009, citado por Martínez y Márquez, 2014) el desarrollo de habilidades investigativas es una de las vías que permite integrar el conocimiento, a la vez sirve como sustento de autoaprendizaje constante; no solo facilitan la solución de las diversas contradicciones que surgen en el ámbito laboral y científico, sino además, permiten la autocapacitación permanente y la actualización sistemática de los conocimientos, lo cual es un indicador de competitividad en la época moderna.

Al 95\% del nivel de confianza la estrategia feedback influye significativamente en el desarrollo de las habilidades instrumentales y afectivas de los estudiantes $(0,00<0,05)$. Existe diferencia significativa en desarrollo de habilidades instrumentales y actitudinales. Los estudiantes lograron manejo óptimo de la tecnológico de la ofimática el software SPSS, comunicación escrita según normas internacionales de redacción y comunicación oral; mayor porcentaje de los estudiantes lograron satisfactoria y excelente en el manejo de trabajo en equipo, aprendizaje colaborativo, responsabilidad e interés de aprendizaje en los temas de investigación científica.

Para Schiavo (2007), las TIC se usa como herramienta en la aplicación de las investigaciones y el tipo de conocimientos. Las TIC constituye actualmente un campo transversal para el trabajo de investigación científica, aportan conocimientos para comprender los procesos de cambio y de construcción social.

Según Hernández y Gallardo (2006), el desarrollo de las habilidades instrumentales investigativas es la reflexión sobre las actividades de investigación que promuevan actitudes de innovación, actualización tecnológica y autoaprendizaje en el educando.

Según Lanchipa (2009), se evidencia influencia significativa del método investigativo en el mejoramiento del nivel de desarrollo de habilidades cognitivas para realizar con éxito las 
tareas de investigación ha demostrado efectividad para contribuir a un mejor desarrollo de habilidades intelectuales para investigar, como habilidades para interpretar información y recoger y organizar información son las deficitarias y mayor uso de técnicas de trabajo grupal.

\section{Conclusión}

Al 95\% de nivel de confianza, la estrategia feedback incide significativamente en el desarrollo de habilidades investigativas de los estudiantes de educación primaria y secundaria de la Facultad de Ciencias de la Educación, 2019 (0,000<0,05). Existe diferencia significativa en el desarrollo de habilidades investigativas, cognitivas, instrumentales y afectivas.

Al 95\% del nivel de confianza la estrategia feedback influye significativamente en el desarrollo de las habilidades cognitivas de los estudiantes $(0,00<0,05)$. Existe diferencia significativa en el manejo de habilidades investigativas cognitivas. Los estudiantes lograron el manejo de la búsqueda de información a través de libros físicos y digitales, revistas científicas electrónicas, distingue evidencias científicas de otro tipo de evidencias empíricas y toma posición del enfoque teórico. Logró con satisfacción el manejo metodológico de la investigación, en el planteamiento del problema, realizar diseño metodológico contextualizado según el enfoque de la investigación y discusión de resultados y conclusión científica en el trabajo.

Al 95\% del nivel de confianza la estrategia feedback influye significativamente en el desarrollo de las habilidades instrumentales y afectivas de los estudiantes $(0,00<0,05)$. Existe diferencia significativa en desarrollo de habilidades instrumentales y actitudinales. Los estudiantes lograron manejo óptimo de la tecnológico de la ofimática el software SPSS, comunicación escrita según normas internacionales de redacción y comunicación oral; mayor porcentaje de los estudiantes lograron satisfactoria y excelente en el manejo de trabajo en equipo, aprendizaje colaborativo, responsabilidad e interés de aprendizaje en los temas de investigación científica. 
Aguilar, E., Rodríguez, A. y Nina, L.B. (2016). La retroalimentación constructiva en el desarrollo de habilidades comunicativas escritas e investigativas en dos generaciones de alumnos de medicina en Yucatán, México, An Fec Med. 77(2), 133-42. ttp://dx.doi.org/10.15381/anales. v77i2.11818

Calderon, R.L. (2010). Influencia del Feedback laboral en el aprendizaje organizacional del personal de la Dirección Regional Sectorial de Comercio Exterior y Turismo de Tacna, 2010. Tesis de Maestría en la Universidad de Jorge Basadre Grohmann de Tacna.

Flores, H. (2015). Situación académica en la UNSCH. Correo, diario local de fecha, o2 de noviembre. https://dialnet.unirioja.es/servlet/articulo?codigo=5236977

Fiallo, J. (2001). La interdisciplinariedad en el currículo: ¿Utopía o Realidad Educativa?. Instituto Central de Ciencias Pedagógicas.

Hernández y Gallardo, S. (2006). Procesos educativos y de investigación en la virtualidad. Suv-UdeG.

Jiménez, F. (2015). Uso del feedback como estrategia de evaluación: aportes desde un enfoque socio constructivista. Actualidades investigativas en educación, 15(1), 1-24. http://dx.doi. org/10.15517/aie.v15i1.17633

Machado, E. y Montes de Oca, N. (2009. Las habilidades investigativas y la nueva Universidad: Terminus a quo a la polémica y la discusión. Humanidades Médicas, 9 (1). http://scielo.sld.cu/ scielo.php?pid=S1727-81202009000100002\&script=sci_arttext

Mamani, D.E. (2018). El feedback aplicado por los formadores de natación en las academias de la ciudad de puno 2018. Tesis de maestría en la Universidad Nacional de Altiplano de Puno. http://repositorio.unap.edu.pe/handle/UNAP/8183

Martínez y Márquez (2014). Las habilidades investigativas como eje transversal de la formación para la investigación. Trabajo de investigación en la Universidad del Pinar del Río de Cuba. file:///C:/Users/Pedro/Downloads/Dialnet-LasHabilidadesInvestigativasComoEjeTransver salDeLa-5236977.pdf

Pérez, C. y López, L. (1999). Las habilidades e invariantes investigativas en la formación del profesorado. Una propuesta metodológica para su estudio. Pedagogía Universitaria, 4(2), 13-44. http://revistas.mes.edu.cu/PedagogiaUniversitaria/articulos/1999/2/189499202.pdf.

Lanchipa, C.F. (2009). Método investigativo y desarrollo de habilidades para investigar en estudiantes de la Escuela de Medicina Humana de la Universidad Nacional Jorge

Basadre Grohmann de Tacna. Tesis de maestría en la Universidad Nacional Mayor de San Marcos.

Schiavo, E. (2007). Investigación científica y tecnológica en el campo de las TIC: ¿conocimientos técnicos, contextuales o transversales?. https://www.redalyc.org/pdf/924/92430907.pdf

(C) Los autores. Este artículo es publicado por la Horizonte de la Ciencia de la Unidad de Posgrado de la Facultad de Educación de la Universidad Nacional del Centro del Perú. Este es un artículo de acceso abierto, distribuido bajo los términos de la Licencia Atribución-No Comercial 4.0 Internacional.(https://creativecommons.org/licenses/by-nc/4.o/), que permite el uso no comercial y distribución en cualquier medio, siempre que la obra original sea debidamente citada. 